\title{
Virgin olive oil and its phenol fraction modulate monocyte/macrophage functionality: a potential therapeutic strategy in the treatment of systemic lupus erythematosus
}

\author{
Marina Aparicio-Soto ${ }^{1} \dagger$, Sergio Montserrat-de la Paz ${ }^{2,3} \dagger$, Marina Sanchez-Hidalgo ${ }^{1}$, Ana Cardeno ${ }^{1}$, \\ Beatriz Bermudez ${ }^{1,4}$, Francisco J. G. Muriana ${ }^{2}$ and Catalina Alarcon-de-la-Lastra ${ }^{1 *}$ \\ ${ }^{1}$ Department of Pharmacology, Faculty of Pharmacy, University of Seville, Professor Garcia Gonzalez Street 2, \\ 41012 Seville, Spain \\ ${ }^{2}$ Laboratory of Cellular and Molecular Nutrition, Instituto de la Grasa, CSIC, Ctra. de Utrera Km. 1, 41013 Seville, Spain \\ ${ }^{3}$ Department of Medical Biochemistry, Molecular Biology and Immunology, School of Medicine, University of Seville, Avda. \\ Dr Fedriani 3, 41071 Seville, Spain \\ ${ }^{4}$ Department of Cell Biology, Faculty of Biology, University of Seville, Avda. Reina Mercedes s/n, 41012 Seville, Spain \\ (Submitted 22 August 2017 - Final revision received 23 May 2018 - Accepted 24 June 2018 - First published online 31 July 2018)
}

\section{Abstract}

Monocytes and macrophages are critical effectors and regulators of inflammation and innate immune response, which appear altered in different autoimmune diseases such as systemic lupus erythematosus (SLE). Recent studies suggested that virgin olive oil (VOO) and particularly its phenol compounds might possess preventive effects on different immune-inflammatory diseases, including SLE. Here, we evaluated the effects of VOO (and sunflower oil) on lipopolysaccharide (LPS)-activated peritoneal macrophages from a model of pristane-induced SLE in BALB/c mice, as well as those of the phenol fraction (PF) from VOO on the immune-inflammatory activity and plasticity in monocytes and monocyte-derived macrophages from healthy volunteers. The release of nitrite and inflammatory cytokines was lower in LPS-treated peritoneal macrophages from pristane-SLE mice fed the VOO diet when compared with the sunflower oil diet. PF from VOO similarly decreased the secretion of nitrite and inflammatory cytokines and expression of inducible nitric oxide, PPAR $\gamma$ and Toll-like receptor 4 in LPS-treated human monocytes. PF from VOO also prevented the deregulation of human monocyte subset distribution by LPS and blocked the genetic signature of M1 macrophages while favouring the phenotype of M2 macrophages upon canonical polarisation of naïve human macrophages. For the first time, our study provides several lines of in vivo and in vitro evidence that VOO and PF from VOO target and counteract inflammatory pathways in the monocyte-macrophage lineage of mice with pristane-induced SLE and of healthy subjects, which is a meaningful foundation for further development and application in preclinical and clinical use of PF from VOO in patients with SLE.

Key words: Virgin olive oil: Dietary phenols: Immunomodulation: Macrophages: Monocytes: Systemic lupus erythematosus

Systemic lupus erythematosus (SLE) is a chronic inflammatory autoimmune disease of unknown aetiology, which affects approximately $0 \cdot 1 \%$ of the population with a large variation across regions and ethnicity. Its pathogenesis is a combination of genetic, environmental and hormonal factors that lead to loss of balance control of cellular immune regulation ${ }^{(1)}$. To date, the majority of studies conducted to understand the pathophysiology of this condition have focused on the auto-reactive $\mathrm{B}$ and $\mathrm{T}$ lymphocytes $^{(2)}$. However, recently, attention has shifted to the role of the innate immune system, and particularly myeloid cells, in this disease. Monocytes/macrophages are critical effectors and regulators of many organ systems, including systemic metabolism, haematopoiesis, angiogenesis and malignancy ${ }^{(3,4)}$. Both monocytes and macrophages are phenotypically altered in SLE; for example, macrophages show reduced uptake of apoptotic cells and enhanced activator status, with altered skew to a proinflammatory direction and an over-production of inflammatory cytokines such as TNF- $\alpha$, IL- 6 and IL- $1 \beta^{(5-7)}$.

Several SLE animal models have been established to investigate SLE disease mechanisms. Among them, pristane

Abbreviations: CCR2, C-C chemokine receptor type 2; iNOS, inducible nitric oxide synthase; LPS, lipopolysaccharide; PF, phenol fraction; RPMI, Roswell Park Memorial Institute; SLE, systemic lupus erythematosus; SOD, diet containing sunflower oil; TLR4, Toll-like receptor 4; VOO, virgin olive oil; VOOD, diet containing virgin olive oil.

* Corresponding author: Professor Dr C. Alarcon-de-la-Lastra, fax +34954556074, email calarcon@us.es

$\dagger$ These authors contributed equally to this work. 
(2,6,10,14-tetramethylpentadecane)-SLE model in BALB/c mice is widely used to test potential therapeutic agents ${ }^{(8,9)}$ as it appears to mimic human idiopathic SLE syndrome closer than spontaneous strains ${ }^{(10)}$. However, despite intensive research, no therapy to date has been found to cure SLE, and current treatments try to control signs and symptoms preventing the damage caused by disease activity and drugs.

In recent years, considerable interest has been given to the ability of diet and different nutritional factors for improving several immune-inflammatory diseases ${ }^{(11)}$. There is broad evidence that dietary therapy can be helpful in the management of SLE symptoms owing to its prophylactic effects without the side effects of classical pharmacology, thus contributing to reduce co-morbidities and to improve health and quality of life of patients with SLE. Current studies emphasised the importance of a diet with plenty of vitamin-rich foods and adequate supply of MUFA/PUFA and dietary fibre, with sodium restriction and moderate energy consumption. The potential contribution of phenols included in the diet for the management of SLE is also noteworthy ${ }^{(12)}$.

The consumption of virgin olive oil (VOO), the major source of MUFA in the traditional Mediterranean diet, is associated with a reduced risk of various chronic inflammatory pathologies ${ }^{(13,14)}$. Many of the preventive properties of VOO have been previously ascribed to its high content of oleic acid. However, it is now generally recognised that minor compounds of VOO, such as the phenol fraction (PF), have also a biological relevance. In this regard, the high concentration of $\mathrm{PF}$ in VOO may contribute in concert with oleic acid to the health benefits of the Mediterranean diet, showing anti-inflammatory, antioxidant and anti-proliferative activities, as well as the ability to modulate relevant cellular signalling pathways ${ }^{(15-17)}$. A diet containing VOO has been shown to be effective in the prevention of kidney damage in mice with pristane-induced SLE and of abnormalities in other mouse models of immune-inflammatory diseases, including rheumatoid arthritis and ulcerative colitis ${ }^{(18-20)}$. Recent studies reported that PF from VOO exerts immune-regulatory activity in vitro in peripheral blood mononuclear cells (PBMC) from patients with SLE and in peritoneal macrophages from wild-type mice ${ }^{(16,21)}$. However, the potential in vivo effects of VOO on inflammation in SLE and in vitro effects of $\mathrm{PF}$ from $\mathrm{VOO}$ in modulating the functional state of the monocyte-macrophage lineage have not been fully investigated.

This study was designed to evaluate the impact of a diet containing VOO on the inflammatory response in peritoneal macrophages from mice with pristane-induced SLE and of PF from VOO on the immune-inflammatory activity and plasticity of human primary monocytes and monocyte-derived macrophages.

\section{Methods}

\section{Animals and diets}

A total of sixty 11- to 12-week-old female BALB/c mice (17 (SEM 2) g) were obtained (Harlan Interfauna Iberica) and maintained in the Animal Laboratory Centre of University of Seville under standard conditions: temperature, $24-25^{\circ} \mathrm{C}$; humidity, $70-75 \%$; and lighting regimen of $12 \mathrm{~h}$ light $-12 \mathrm{~h}$ dark cycle. They were fed standard rodent chow (Panlab A04) and water ad libitum until pristane induction of SLE-like disease. Experimental diets were formulated on the basis of the American Institute of Nutrition standard reference diet with the modification of varying sources of carbohydrates and the principal source for fats (10\% sunflower oil or VOO) (online Supplementary Table S1).

\section{Pristane-systemic lupus erythematosus model and mouse peritoneal macrophages}

At 3 months of age, SLE was induced in half of the animals by means of an intraperitoneal injection of $0.5 \mathrm{ml}$ of pristane $(99 \%$ pure; Sigma-Aldrich) according to the procedure described by Satoh \& Reeves ${ }^{(8)}$. The other half of the animals were subjected to an intraperitoneal injection of saline solution.

Mice were then randomised into the following four experimental groups (fifteen animals per group): (1) animals injected with saline solution and fed a diet containing a marketable sunflower oil (Koipesol-Deoleo) (SOD); (2) animals treated with pristane and fed SOD; (3) animals injected with saline solution and fed a diet containing a marketable VOO (Olea europaea L., picual variety; Oleoestepa SAC) (VOOD); and (4) animals treated with pristane and fed VOOD. During the entire duration of the experiment, mortality, weight and water and food consumption were monitored weekly. The composition of experimental diets and their content of fatty acids, sterols, squalene, triterpenic alcohols and tocopherols is available in online Supplementary Table S2.

After 24 weeks of the experimental period, animals were euthanised by overdoses of pentobarbital. Peritoneal exudate cells (macrophages) were then harvested by washing the peritoneal cavity with sterile ice-cold PBS according to the protocol described by Aparicio-Soto et $a l^{(22)}$. In brief, after centrifugation, cells were resuspended in Roswell Park Memorial Institute (RPMI) 1640 medium supplemented with L-glutamine $(2 \mathrm{~mm})$, glucose $(4.5 \mathrm{~g} / \mathrm{l})$, heat-inactivated fetal calf serum (FCS, $10 \%$ ), penicillin $(100 \mathrm{U} / \mathrm{ml})$, streptomycin $(100 \mathrm{mg} / \mathrm{ml})$ and HEPES (10 mm) (PAA Laboratories $\mathrm{GmbH}$ ), and then seeded in culture plates $\left(1 \times 10^{6}\right.$ cells $\left./ \mathrm{ml}\right)$ for $2 \mathrm{~h}$ at $37^{\circ} \mathrm{C}$ in a $5 \% \mathrm{CO}_{2}$ humidified atmosphere. Non-adherent cells were removed by washing with PBS, and fresh RPMI 1640 medium supplemented with FCS (5\%) was added to cultured peritoneal macrophages for further experimentation (see below).

All animal care and experimental procedures were performed according to a protocol approved by the Animal Ethics Committee of the University of Seville, and all experiments were in accordance with the recommendations of the European Union regarding animal experimentation (Directive of the European Counsel 2010/630/EU) and Animal Research: Reporting of In Vivo Experiments (ARRIVE) guidelines for reporting experiments involving animals ${ }^{(23,24)}$.

\section{Human monocytes and their differentiation into macrophages}

Healthy volunteers were recruited at Virgen del Rocio University Hospital for peripheral blood collection and isolation of 
monocytes. The study conformed to the principles outlined in the Helsinki Declaration of the World Medical Association. Donors (adult $<35$ years old) were non-smokers and not taking any medication. They were recognised as healthy according to medical history and routine laboratory test. Blood samples were collected in $\mathrm{K}_{3}$ EDTA-containing Vacutainer tubes (Becton Dickinson), and PBMC were isolated by centrifugation over Ficoll Histopaque gradient (Sigma-Aldrich). Monocytes were isolated from PBMC using positive selection with CD14 MicroBeads according to the manufacturer's instructions (MACS; Myltenyi Biotec). The purity of monocytes was tested by CD14 fluorescein isothiocyanate labelling and fluorescenceactivated cell sorting (FACS) analysis using a FACScanto II flow cytometer (BD Biosciences). After isolation, cells were suspended in RPMI 1640 medium supplemented with L-glutamine, penicillin, streptomycin and heat-inactivated FCS (10\%) at a density of $5 \times 10^{5}$ cells $/ \mathrm{ml}$.

Monocytes were induced to differentiate during $6 \mathrm{~d}$ in RPMI 1640 supplemented with L-glutamine, penicillin, streptomycin, heat-inactivated FCS (10\%) and recombinant human macrophage colony-stimulating factor (rhM-CSF, $25 \mathrm{ng} / \mathrm{ml}$ ) to obtain naïve M0 macrophages. Every $2 \mathrm{~d}$, fresh medium containing rhM-CSF was added. M0 macrophages were then exposed (for additional $24 \mathrm{~h}$ ) to lipopolysaccharide (LPS) (100 ng/ml) and interferon (IFN)- $\gamma(20 \mathrm{ng} / \mathrm{ml})$ for M1 polarisation or to IL-4 $(20 \mathrm{ng} / \mathrm{ml})$ for $\mathrm{M} 2$ polarisation in the presence or absence of PF from $\operatorname{VOO}(25$ and $50 \mu \mathrm{g} / \mathrm{ml})$.

\section{Extraction and chemical characterisation of phenol fraction from virgin olive oil}

The same VOO used above for animal diets was used to extract PF according to the procedure described by Vazquez Roncero et al. $^{(26)}$, with some modifications ${ }^{(16)}$. Quantitative and qualitative analysis of PF was performed according to COI/T20/ 29doc (International Olive Council) by high-performance ternary gradient liquid chromatography. The content of total phenols was calculated by measuring the sum of the areas of the related chromatographic peaks and expressed in $\mathrm{mg} / \mathrm{kg}$ of tyrosol. The composition of the isolated $\mathrm{PF}$ from $\mathrm{VOO}$ is detailed in Table 1.

\section{Treatments with lipopolysaccharide}

Mouse peritoneal macrophages were treated with $5 \mu \mathrm{g} / \mathrm{ml}$ LPS in the presence or absence of PF ( 25 or $50 \mu \mathrm{g} / \mathrm{ml}$ ) diluted in dimethylsulphoxide for $18 \mathrm{~h}$. In the case of human monocytes/ macrophages, the concentration of LPS was $100 \mathrm{ng} / \mathrm{ml}$ and the incubation time was $24 \mathrm{~h}$.

\section{Measurement of nitrite production}

Cell culture supernatants were transferred to a ninety-six-well assay plate and mixed with Griess reagent (Sigma-Aldrich). The amount of nitrite, as an index of NO generation, was determined by a spectrophotometric method according to the Griess reaction $^{(26)}$ and by extrapolation from a standard curve with sodium nitrite as standard. The absorbance at $540 \mathrm{~nm}$ was measured by using a microtitre plate reader (BioTek).

\section{Cytokine assay}

The concentration of IL- $1 \beta$, IL- 6 , IL-17 and TNF- $\alpha$ in cell culture supernatants was determined using appropriate commercial ELISA kits (Diaclone). Values were expressed in $\mathrm{pg} / \mathrm{ml}$, as calculated from calibration curves after serial dilutions of human recombinant standards for each assay. The intensity of each sample was read at $450 \mathrm{~nm}$ in a microtitre plate reader.

\section{Cell viability assay}

Cells seeded in ninety-six-well plates $\left(1 \times 10^{5}\right.$ cells/well $)$ were incubated in the presence or absence of PF for $24 \mathrm{~h}$. At the end of the exposure time, the effect on cell growth/viability was analysed by the mitochondrial-dependent reduction of 3-(4,5dimethylthiazol-2-yl)-2,5-diphenyltetrazolium bromide to formazan (Sigma-Aldrich) ${ }^{(27)}$. Cell survival was measured as the percentage of absorbance compared with that obtained in control, non-treated cells.

\section{RNA isolation and real-time quantitative $P C R$ analysis}

Total RNA was extracted from cells by using TRIsure reagent (Bioline), as instructed by the manufacturer. RNA quality was

Table 1. Composition of the phenol extract (PF) from virgin olive oil using COI/T20/29doc

\begin{tabular}{|c|c|c|c|}
\hline Phenol names & Percentage & Amount ( $\mu \mathrm{g}$ of phenols in $50 \mu \mathrm{g}$ PF) & Concentration ( $\mu \mathrm{m}$ of phenols in $50 \mu \mathrm{g} \mathrm{PF} / \mathrm{ml}$ ) \\
\hline Hydroxytyrosol & $12 \cdot 85$ & 6.43 & $41 \cdot 71$ \\
\hline Tyrosol & 12.69 & $6 \cdot 34$ & $45 \cdot 94$ \\
\hline Vanillic acid & 1.74 & 0.87 & $5 \cdot 17$ \\
\hline$p$-Coumaric acid & $1 \cdot 14$ & 0.57 & 3.47 \\
\hline Decarboxymethyl oleuropein aglycone (dialdehyde) & 6.69 & 3.34 & $10 \cdot 43$ \\
\hline Tyrosyl acetate & 1.83 & 0.91 & 5.05 \\
\hline Decarboxymethyl ligstroside aglycone (dialdehyde) & $7 \cdot 00$ & 3.50 & 11.51 \\
\hline Pinoresinol & 4.49 & $2 \cdot 24$ & $6 \cdot 25$ \\
\hline Cinnamic acid & 1.94 & 0.97 & 6.54 \\
\hline Acetoxy-pinoresinol & $5 \cdot 26$ & 2.63 & $6 \cdot 32$ \\
\hline Oleuropein aglycone, aldehyde form & $25 \cdot 20$ & $12 \cdot 60$ & $39 \cdot 37$ \\
\hline Ligstroside aglycone, dialdehyde form & $16 \cdot 17$ & 8.09 & $26 \cdot 61$ \\
\hline Luteolin & 2.49 & $1 \cdot 24$ & 4.33 \\
\hline Apigenin & 0.49 & 0.24 & 0.89 \\
\hline Total phenols expressed in tyrosol & $600 \mathrm{mg} / \mathrm{kg}$ & & \\
\hline
\end{tabular}


assessed by $A_{260}: A_{280}$ ratio in a NanoDrop ND-1000 Spectrophotometer (Thermo Scientific). RNA ( $1 \mu \mathrm{g})$ was subjected to reverse transcription (iScript; Bio-Rad) according to the manufacturers' protocol. An amount of $20 \mathrm{ng}$ of the resulting complementary DNA (cDNA) was used as a template for real-time PCR amplifications. The mRNA levels for specific genes were determined by real-time PCR in a MX3000P system (Stratagene). For each PCR reaction, cDNA template was added to Brilliant SYBR green QPCR Supermix (Bio-Rad) containing the primer pairs for either gene or for glyceraldehyde 3-phosphate dehydrogenase $(G A P D H)$ and $18 \mathrm{~S}$ ribosomal (18S) as housekeeping genes. The sequence and additional information for the primers used are in online Supplementary Table S3. All amplification reactions were performed in triplicate, and average threshold cycle $\left(C_{\mathrm{t}}\right)$ numbers of the triplicates were used to calculate the relative mRNA expression of candidate genes. The magnitude of change of mRNA expression for candidate genes was calculated by using the standard $2^{-\Delta \Delta C_{t}}$ method. All data were normalised to endogenous reference (GAPDH and 18S) gene content and expressed as fold of controls.

\section{Flow cytometry analysis}

Surface membrane expression of CD14 (PE anti-human CD14; Miltenyi Biotec), CD68 (FITC anti-human CD68; Miltenyi Biotec), CD16 (APC-Cy7 anti-human CD16; Miltenyi Biotec) and C-C chemokine receptor type 2 (CCR2) (APC anti-human CCR2; BD Biosciences) on monocytes was assessed by FACS analysis. According to the manufacturer's instructions, $5 \times 10^{5}$ purified monocytes after in vitro treatment in the presence or absence of LPS were incubated with antibodies at room temperature, in the dark, for $15 \mathrm{~min}$, followed by fixation and lysing of erythrocytes with $20 \times$ volume of FACS lysing solution (BD Biosciences). Fluorescence intensity was measured by a FACScanto II flow cytometer and calibrated using CellQuest ${ }^{\mathrm{TM}}$ software (BD Biosciences). Results were analysed using the Win-List software package (Verity Software House). Mean fluorescence intensity (MFI) of $10^{4}$ counted cells was measured in each sample. Monocytes were gated as forward scatter ${ }^{\text {high }}$ (FSC $\left.{ }^{\text {high }}\right)$-side scatter ${ }^{\text {high }}$ $\left(\mathrm{SSC}^{\text {high }}\right)$ cells. Expression levels were presented as MFI corrected for non-specific binding of isotype control antibodies.

\section{Isolation and immunoblotting detection of proteins}

Cells were rinsed, collected and processed as described by Sanchez-Hidalgo et $a l .{ }^{(28)}$. Protein concentration was measured for each sample using a protein assay reagent (Bio-Rad) according to Bradford's method using $\gamma$-globulin as a standard $^{(29)}$. Aliquots of supernatant containing equal amount of proteins $(20 \mu \mathrm{g})$ were separated on $10 \%$ acrylamide gel by SDSPAGE, and the proteins were electrophoretically transferred into a nitrocellulose membrane and incubated with specific primary antibodies, such as polyclonal mouse anti-human PPAR $\gamma$ (Abcam), rabbit anti-inducible nitric oxide synthase (iNOS) (Cayman Chemical) (1:100 000) and monoclonal mouse anti-human $\beta$-actin (Sigma-Aldrich) antibodies, overnight at $4^{\circ} \mathrm{C}$. After rinsing, the membranes were incubated with a horseradish peroxidase-labelled secondary antibody anti-rabbit (Cayman
Chemical) (1:50 000) or anti-mouse (Dako) (1:2000) containing blocking solution for $1-2 \mathrm{~h}$ at room temperature. Immunodetection was performed using enhanced chemiluminescence light-detecting kit (Pierce). The signals were captured using an LAS-3000 Imaging System (Fujifilm), and densitometry data were studied following normalisation to the housekeeping loading control. The signals were analysed and quantified by Image Processing and Analysis in Java (Image J; Softonic) and expressed in relation to LPS-treated cells.

\section{Statistical analysis}

All values in the figures and text are expressed as arithmetic means with their standard errors. Experiments were carried out in triplicate. Data were evaluated with GraphPad Prism version 6.01 software. The statistical significance of any difference in each parameter among the groups was evaluated by one-way ANOVA using Tukey's multiple comparisons test as post hoc test. $P$ values $<0.05$ were considered statistically significant. In the experiments involving densitometry, figures are representative of at least three different experiments performed on different days.

\section{Results}

Effects of diet containing sunflower oil and virgin olive oil on nitrite production in peritoneal macrophages from pristane-systemic lupus erythematosus mice

As shown in Fig. 1(a), nitrite production was induced in peritoneal macrophages from pristane-SLE mice when fed SOD but not with VOOD. The in vitro treatment of peritoneal macrophages from vehicle control mice with LPS did not induce nitrite production; however, nitrite was produced in LPS-treated peritoneal macrophages from pristane-SLE mice, with this effect being significantly less pronounced in animals feeding on VOOD when compared with animals feeding on SOD.

\section{Effects of diet containing sunflower oil and virgin olive oil on pro-inflammatory cytokine production in peritoneal macrophages from pristane-systemic lupus erythematosus mice}

The production of IL- 6 , TNF- $\alpha$ and IL-17 was virtually absent in peritoneal macrophages from vehicle control mice fed either SOD or VOOD (data not shown). In addition, no significant differences were found for the production of IL-6 (Fig. 1(b)), TNF- $\alpha$ (Fig. 1(c)) and IL-17 (Fig. 1(d)) between LPS-treated peritoneal macrophages from vehicle control mice fed SOD and VOOD. However, the production of these cytokines was increased in LPS-treated peritoneal macrophages from pristane-SLE mice, with this effect being significantly less pronounced in animals feeding on VOOD when compared with animals feeding on SOD.

\section{Effects of phenol fraction from virgin olive oil on viability of human monocytes}

After $24 \mathrm{~h}$ of treatment with PF $(6 \cdot 25-50 \mu \mathrm{M})$, the viability of human monocytes ( $>95 \%$ were alive) was not affected (data not shown). 
Effects of phenol fraction from virgin olive oil on nitrite production and inducible nitric oxide synthase expression in lipopolysaccharide-treated human monocytes

LPS is a strong activator of the inflammatory response and immune regulation, which induces human monocytes to secrete different cytokines, including TNF- $\alpha$ and IL- $1 \beta$, among others $^{(30)}$. As shown in Fig. 2(a), nitrite production was induced in LPS-treated human monocytes. However, this effect was significantly reduced and accompanied by a down-regulation in the protein (Fig. 2(b)) and gene (Fig. 2(c)) expression of iNOS by $\mathrm{PF}$ from VOO in a dose-dependent manner.

\section{Effects of phenol fraction from virgin olive oil on PPAR expression in lipopolysaccharide-treated human monocytes}

While protein (Fig. 2(d)) and gene (Fig. 2(e)) expression of PPAR $\gamma$ was down-regulated in LPS-treated human monocytes, this effect was opposed by PF from VOO, which up-regulated protein and gene expression of PPAR $\gamma$ in a dose-dependent manner.

\section{Effects of phenol fraction from virgin olive oil on} pro-inflammatory cytokine and Toll-like receptor 4 expression in lipopolysaccharide-treated human monocytes

Both secretion (Fig. 3(a)-(c)) and gene expression (Fig. 3(d)-(f)) of IL- 6 , TNF- $\alpha$ and IL- $1 \beta$ were induced in LPS-treated human monocytes. These effects were significantly reduced by PF from VOO in a dose-dependent manner.

Toll-like receptor 4 (TLR4) is extensively expressed in immune cells and plays a critical role in recognition and signalling of bacterial LPS, inducing cytokine release ${ }^{(31,32)}$. TLR4 gene expression (Fig. 3(g)) was induced in LPS-treated human monocytes. However, this effect was significantly reduced by $\mathrm{PF}$ from $\mathrm{VOO}$ in a dose-dependent manner.

\section{Effects of phenol fraction from virgin olive oil on} polarisation in human monocyte-derived macrophages

Monocytes from healthy donors were cultured with rhM-CSF to mature into naïve M0 macrophages. FACS analysis showed that approximately $97 \%$ of M0 macrophages were CD68positive cells (data not shown). Then, cells were treated with canonical stimuli for polarisation into M1 or M2 macrophages in the presence or absence of PF from VOO. In comparison with M1 macrophages with increased prototypical markers (CD80, CD64 and monocyte chemoattractant protein-1 (MCP-1)), those macrophages polarised to M1 in the presence of PF from VOO had a significant decreased gene expression of CD80 (Fig. 4(a)), CD64 (Fig. 4(b)) and MCP-1 (Fig. 4(c)). These effects of PF from VOO appeared to be dosedependent. In addition, the relative gene expression of CD200R (Fig. 4(d)), MRC-1 (Fig. 4(e)) and CD36 (Fig. 4(f)) (as prototypical M2 markers) was significantly increased by $\mathrm{PF}$ from VOO, mainly at high dose $(50 \mu \mathrm{g} / \mathrm{ml})$, after M2 polarisation. The effects of PF from VOO in reducing gene expression of CD80 and MCP-1 in M1-polarised macrophages were not
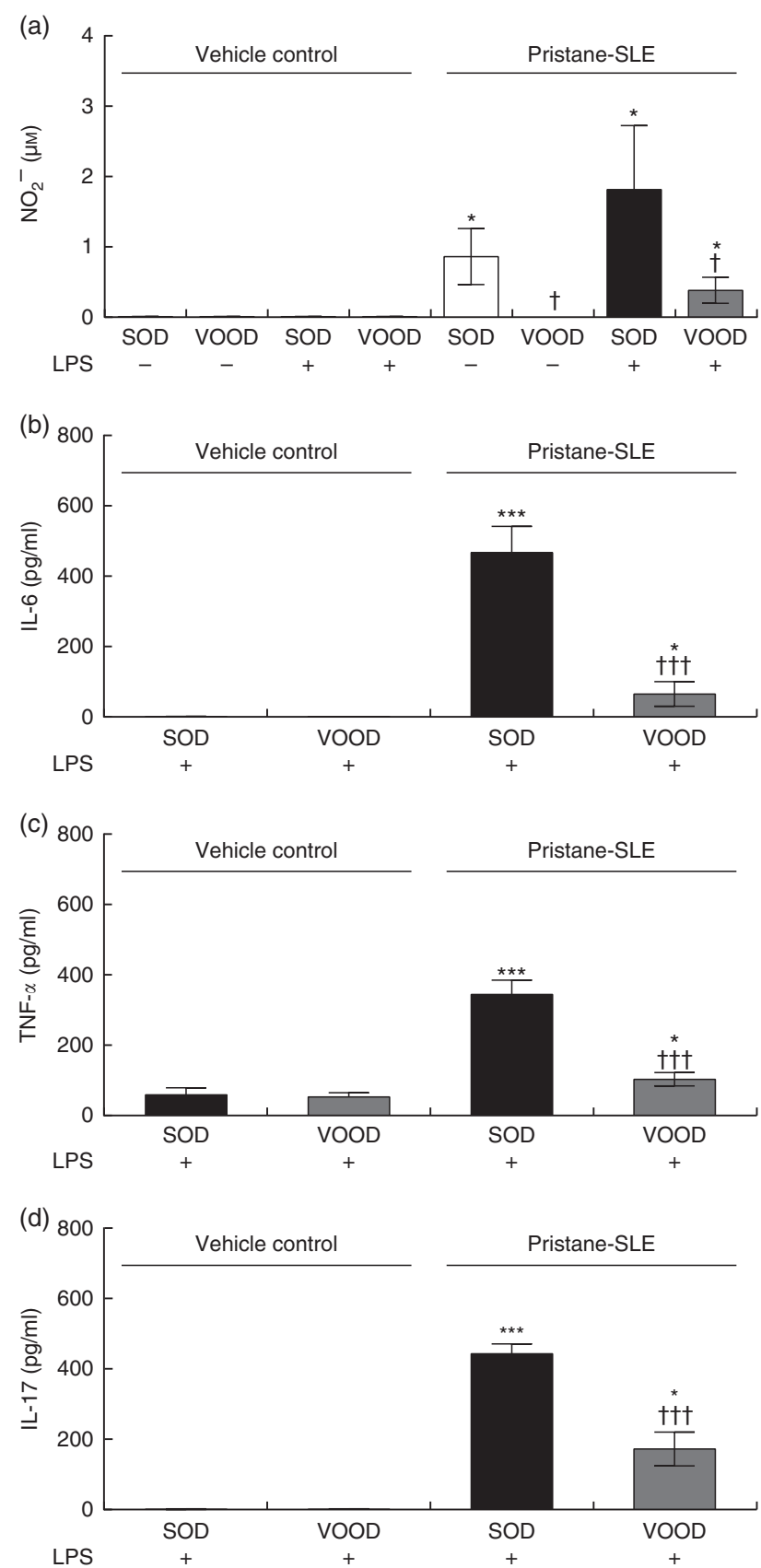

Fig. 1. Sunflower oil and virgin olive oil (VOO) diets on nitrite and proinflammatory cytokine production in lipopolysaccharide (LPS)-treated peritoneal macrophages from pristane-systemic lupus erythematosus (SLE) mice. Animals were injected with saline solution or pristane and then fed a diet containing sunflower oil (SOD) or VOO (VOOD) as indicated. After isolation, peritoneal macrophages were treated or not treated with LPS for $24 \mathrm{~h}$, after which the supernatant was collected. (a) Nitrite, (b) IL-6, (c) TNF- $a$ and (d) IL-17 concentration. Values are means ( $n 10$ per group), with their standard errors represented by vertical bars. ${ }^{*} P<0.05$ and ${ }^{* \star *} P<0.001$ v. vehicle control (saline solution); $† P<0.05$ and $\dagger \dagger \dagger P<0.001$ v. SOD.

comparable to those observed in M2-polarised macrophages; however, PF from VOO potentiated the transcriptional activity of CD200R, MRC-1 and CD36 genes involved in the M2 phenotypic change. 
(a)
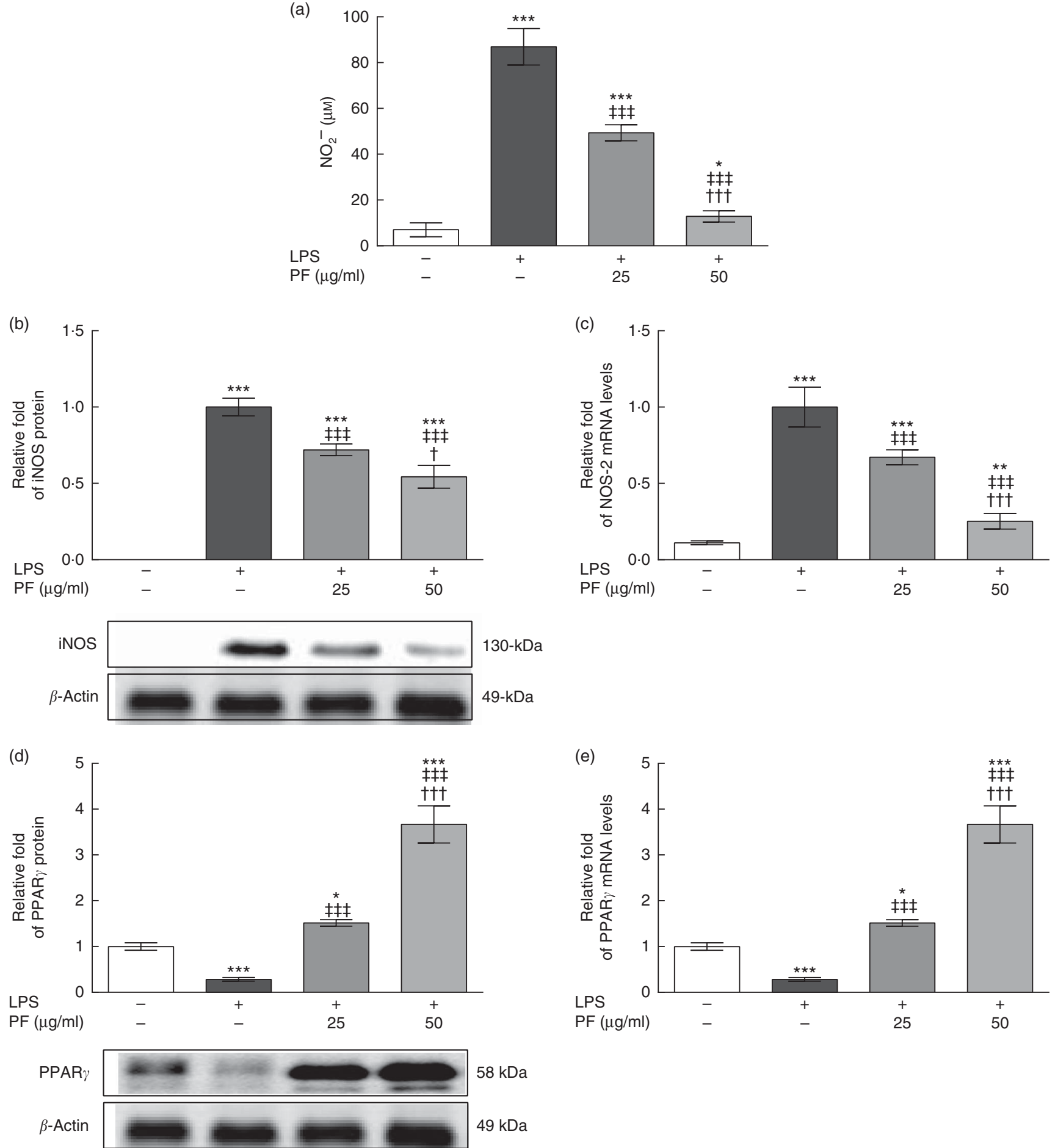

Fig. 2. Phenol fraction (PF) from virgin olive oil (VOO) on nitrite production and inducible nitric oxide synthase (iNOS) and PPAR $y$ expression in lipopolysaccharide (LPS)-treated human monocytes. Cells were isolated from peripheral blood samples of healthy volunteers and immediately treated or not treated with LPS in the presence $(25$ and $50 \mu \mathrm{g} / \mathrm{ml}$ ) or absence of PF from VOO for $24 \mathrm{~h}$, after which the supernatant, cellular proteins and RNA were collected. (a) Nitrite concentration. (b) Relative fold change in band intensity of iNOS protein. (c) Relative fold change in mRNA level of NOS-2 gene. (d) Relative fold change in band intensity of PPARy protein. (e) Relative fold change in mRNA level of PPAR $y$ gene. $\beta$-Actin was served as an equal loading control for normalisation of protein levels. Values are means for three independent experiments in triplicate, with their standard errors represented by vertical bars. ${ }^{*} P<0.05,{ }^{\star *} P<0.01$ and ${ }^{* \star *} P<0.001 \mathrm{~V}$. control non-LPS-treated cells; $\dagger P<0.05$ and $\dagger \dagger \dagger P<0.001 \mathrm{v}$. other PF concentration; 㧊 $P<0.001 \mathrm{v}$. LPS-treated cells. 
(a)
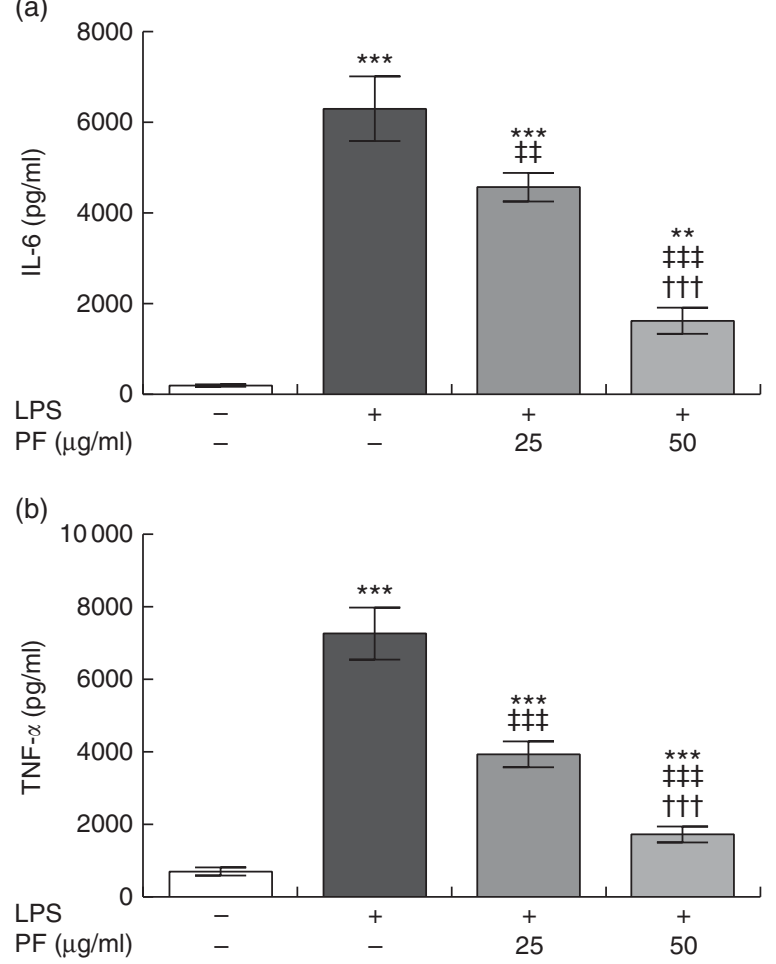

(c)

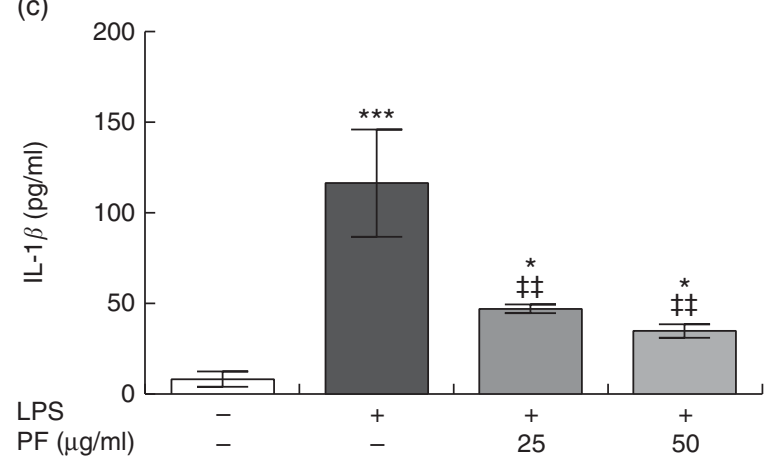

(d)

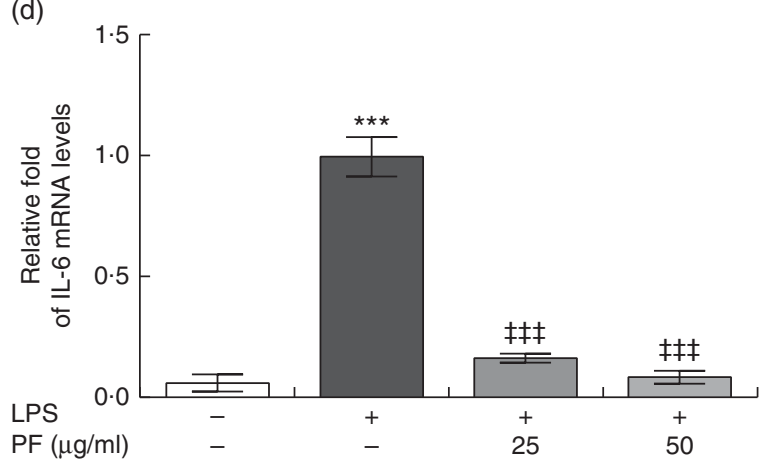

(e)

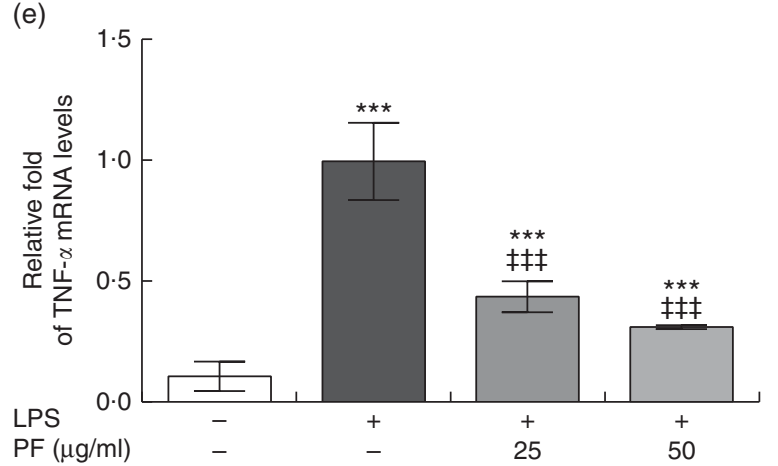

(f)

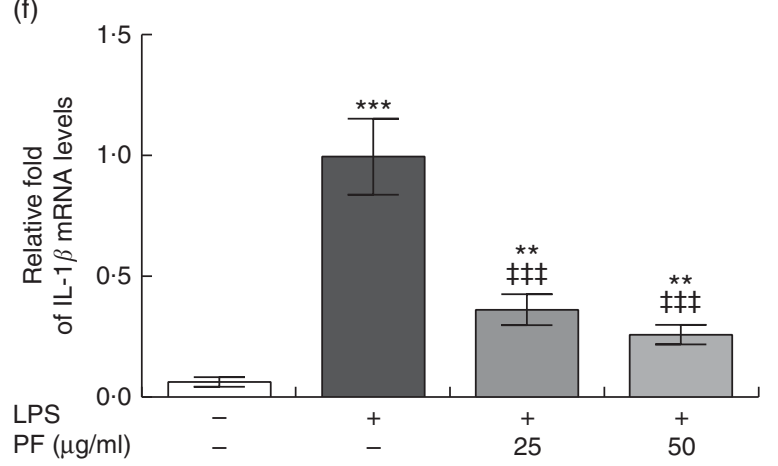

(g)

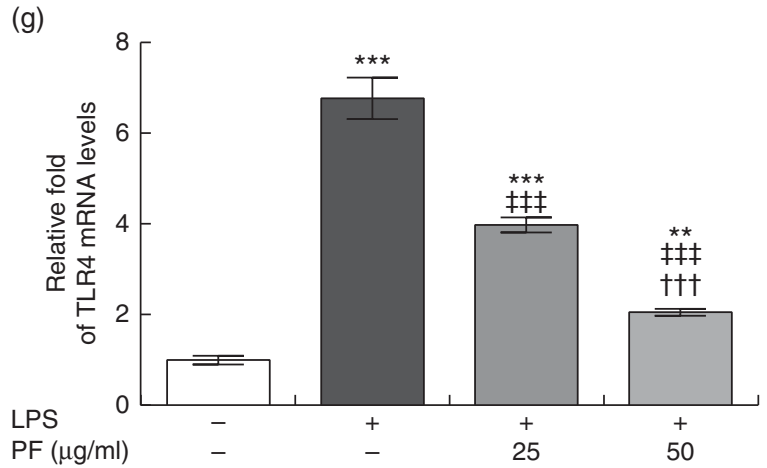

Fig. 3. Phenol fraction (PF) from virgin olive oil (VOO) on pro-inflammatory cytokine production and gene expression, and Toll-like receptor 4 (TLR4) gene expression in lipopolysaccharide (LPS)-treated human monocytes. Cells were isolated from peripheral blood samples of healthy volunteers and immediately treated or not treated with LPS in the presence ( 25 and $50 \mu \mathrm{g} / \mathrm{ml}$ ) or absence of PF from VOO for $24 \mathrm{~h}$, after which the supernatant and cellular RNA were collected. (a) IL-6, (b) TNF- $a$ and (c) IL-1 $\beta$ concentration. $(\mathrm{d}-\mathrm{g})$ Relative fold change in mRNA level of IL-6, TNF- $\alpha, \mathrm{IL}-1 \beta$ and TLR4 genes, respectively. $\beta$-Actin was served as an equal loading control for normalisation of protein levels. Values are means of three independent experiments in triplicate, with their standard errors represented by vertical bars. ${ }^{*} P<0.05$, ${ }^{\star \star} P<0.01$ and ${ }^{\star \star \star} P<0.001 \mathrm{v}$. control non-LPS-treated cells; $\dagger \dagger \dagger P<0.001 \mathrm{v}$. other PF concentration; $\neq \ddagger P<0.01$ and $\ddagger \ddagger \ddagger P<0.001 \mathrm{v}$. LPS-treated cells. 
(a)

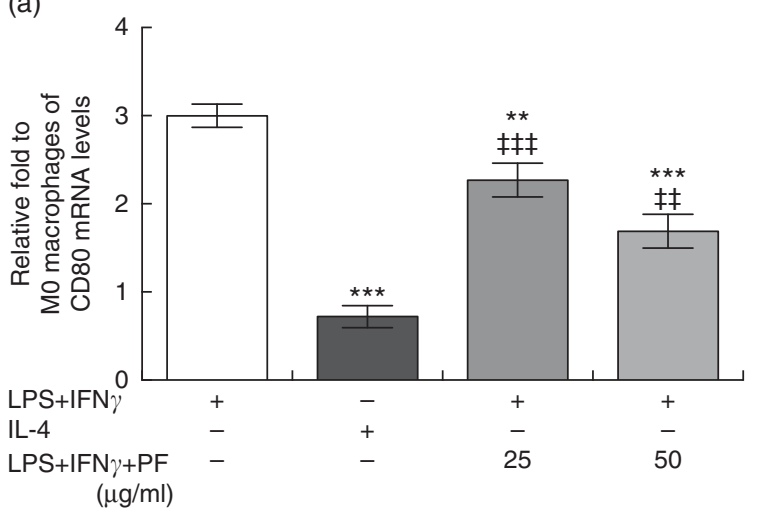

(b)

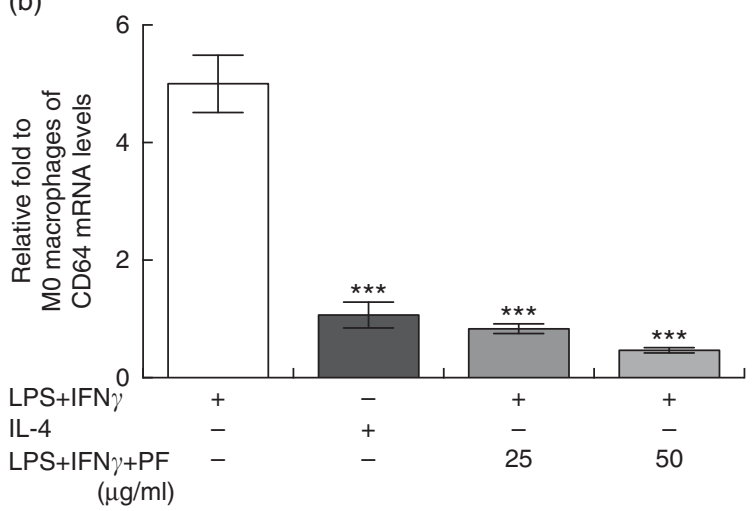

(c)

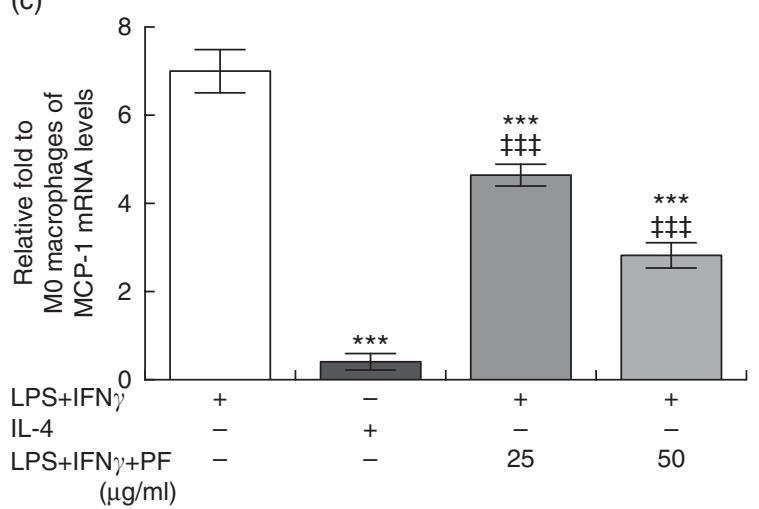

(d)

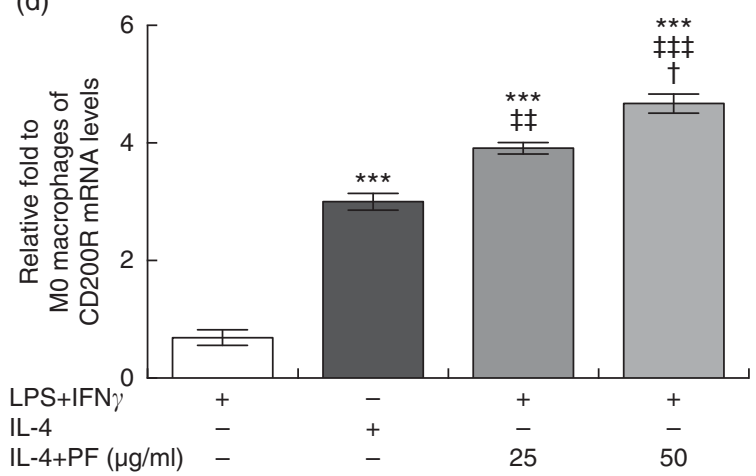

(e)

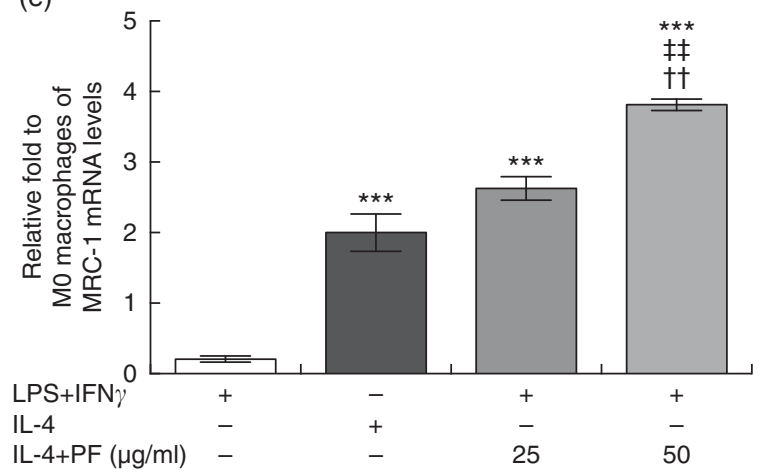

(f)

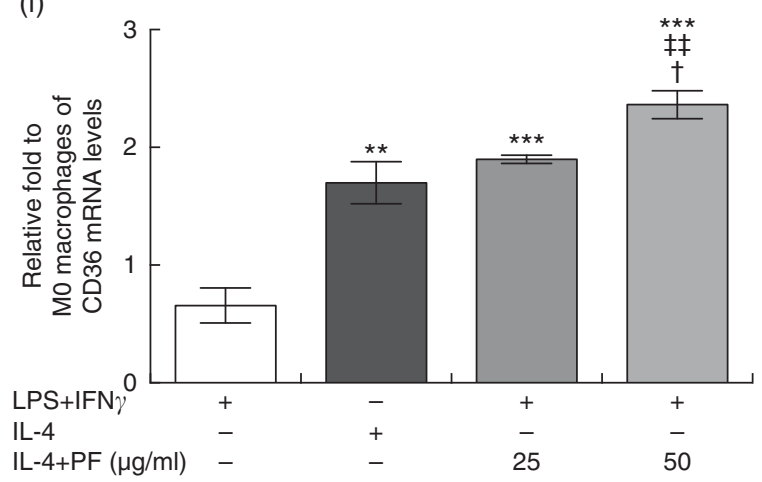

Fig. 4. Phenol fraction (PF) from virgin olive oil (VOO) on polarisation in human monocyte-derived macrophages. Monocytes were isolated from peripheral blood samples of healthy volunteers and immediately cultured with macrophage colony-stimulating factor for $6 \mathrm{~d}$ to differentiate into naïve Mo macrophages. These cells were then treated with lipopolysaccharide (LPS) and interferon (IFN)- $\gamma$ to polarise into M1 or with IL-4 to polarise into M2 macrophages in the presence (25 and $50 \mu \mathrm{g} / \mathrm{ml}$ ) or absence of PF from VOO for $24 \mathrm{~h}$, after which the cellular RNA was collected. (a-c) Relative fold change in mRNA level of CD80, CD64 and MCP-1 genes in M1/M2 compared with M0 macrophages. (d-f) Relative fold change in mRNA level of CD200R, MRC-1 and CD36 genes in M1/M2 compared with M0 macrophages. Values are means for three independent experiments by triplicate, with their standard errors represented by vertical bars. ${ }^{* \star} P<0.01$ and ${ }^{* \star *} P<0.001 \mathrm{v}$. M1 macrophages;

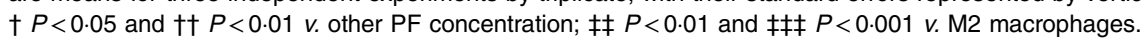

\section{Effects of phenol fraction from virgin olive oil on} distribution of human monocyte subsets during lipopolysaccharide challenge

Human monocyte subsets are based on their expression of cell surface markers CD14 (LPS co-receptor) and CD16 (FC gamma receptor $\mathrm{II})^{(33)}$. Classical monocytes are defined as $\mathrm{CD} 14^{+}$ ${ }^{+} \mathrm{CD} 16^{-}$cells, intermediate monocytes as $\mathrm{CD} 14^{++} \mathrm{CD} 16^{+}$cells and non-classical monocytes as $\mathrm{CD} 14^{+} \mathrm{CD} 16^{++}$cells. Classical monocyte subsets were increased in LPS-treated human monocytes (Fig. 5(a) and (b)). This effect was counteracted by $\mathrm{PF}$ from $\mathrm{VOO}$ in a dose-dependent manner, with a monocyte subset distribution at $50 \mu \mathrm{g} / \mathrm{ml}$ of PF similar to that observed in untreated human monocytes. In agreement, the increased surface expression of the prototypical classical monocyte marker CCR2 in LPS-treated human CD14 $4^{++} \mathrm{CD} 16^{-}$cells (Fig. 5(c)) was less pronounced by $\mathrm{PF}$ from $\mathrm{VOO}$ in a dose-dependent manner. Similar effects of PF from VOO were observed on gene (Fig. 5(d)) and surface (Fig. 5(e)) expression of CCR2 in the entire monocyte population treated with LPS. 
(a)

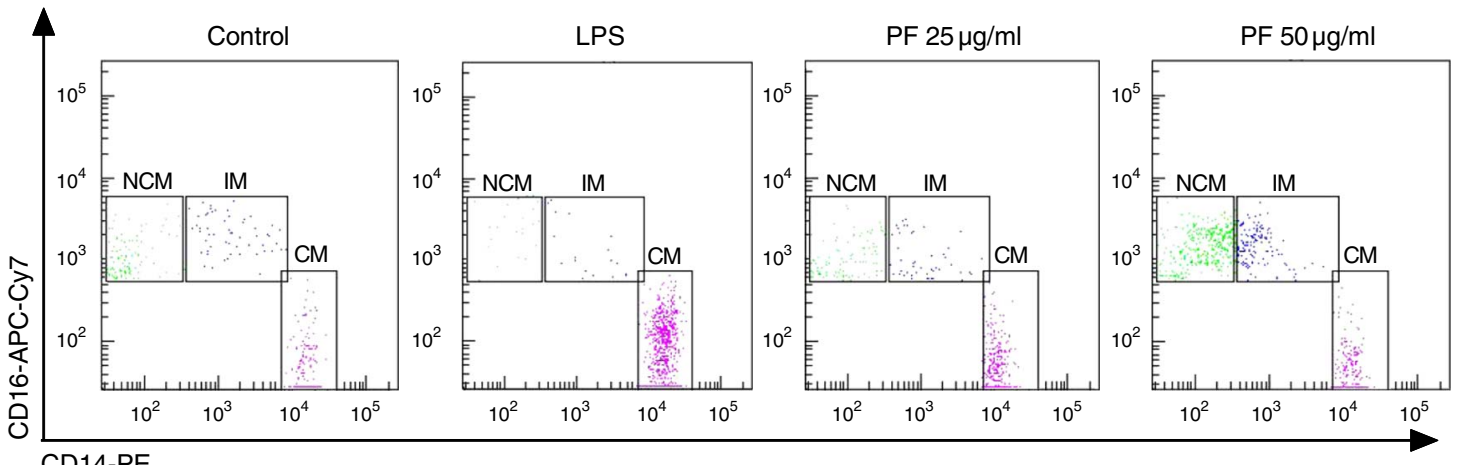

(b)

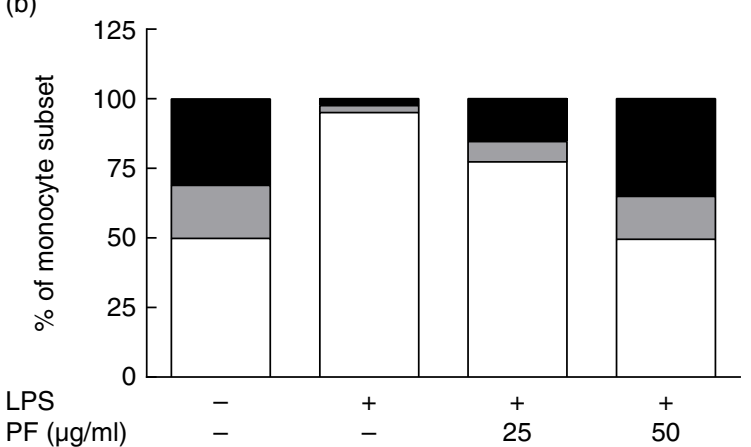

(c)

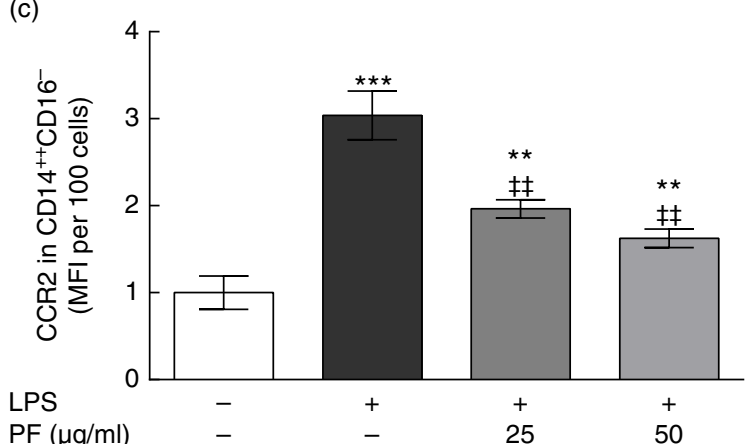

(d)

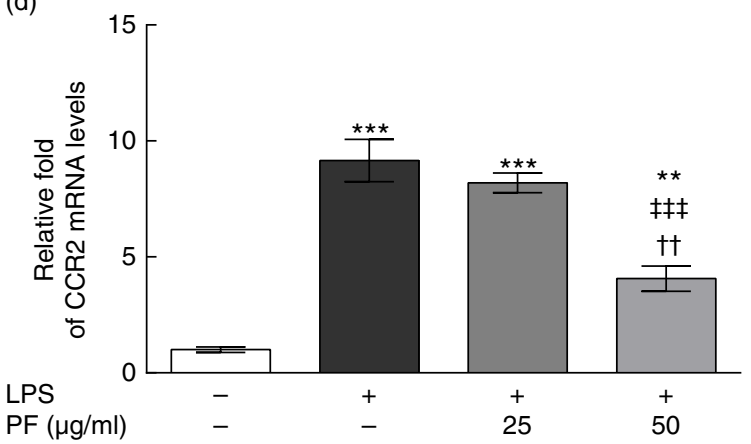

(e)
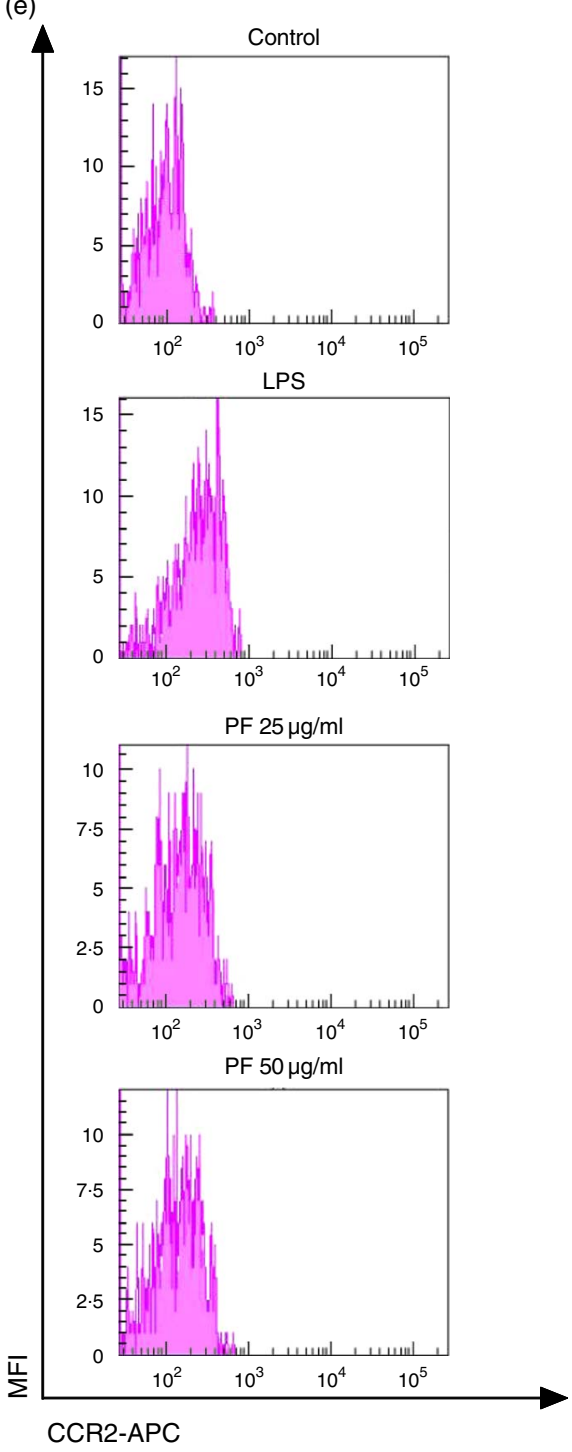

Fig. 5. Phenol fraction (PF) from virgin olive oil (VOO) on distribution of human monocyte subsets during lipopolysaccharide (LPS) challenge. Monocytes were isolated from peripheral blood samples of healthy volunteers and immediately treated or not treated with LPS in the presence ( $25 \mathrm{and} 50 \mu \mathrm{g} / \mathrm{ml})$ or absence of PF from VOO for $24 \mathrm{~h}$, after which they were stained for fluorescence-activated cell sorting analysis of surface markers CD14 and CD16 or the cellular RNA was collected. (a) Flow cytometry analysis of monocyte subsets according to their CD14 and CD16 surface expression (CM, classical monocytes as $C D 14^{++} \mathrm{CD} 16^{-}$cells; IM, intermediate monocytes as CD14 ${ }^{++} \mathrm{CD} 16^{+}$ cells; NCM, non-classical monocytes as CD14 ${ }^{+} \mathrm{CD} 16^{++}$cells). (b) Percentage of classical $(\square)$, intermediate $(\square)$ and non-classical ( $\square$ ) monocytes. (c) C-C chemokine receptor type 2 (CCR2) surface expression in classical, $C D 14^{++} \mathrm{CD} 16^{-}$monocytes. (d) Relative fold change in mRNA level of CCR2 gene. (e) Representative flow cytometry plots of CCR2 surface expression. Values are means of three independent experiments in triplicate, with their standard errors represented by vertical bars. ${ }^{* *} P<0.01$ and

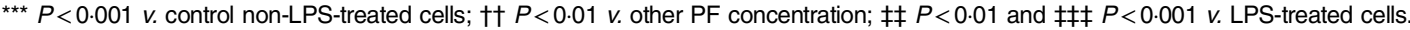




\section{Discussion}

SLE is an autoimmune disease characterised by autoantibody production and chronic inflammation in multiple organs, whose aetiology still remains unclear. In the past, most of the SLE pathogenesis studies considered the adaptive immune system to be the primary cause of autoimmunity, focusing on the primary abnormalities of $\mathrm{B}$ and $\mathrm{T}$ lymphocyte functions. More recent studies are now centred on the innate immunity and on how the SLE autoimmune response is initiated and maintained ${ }^{(34)}$. Of note, monocytes and macrophages play a pivotal role in the innate immune system with widespread immunological function $^{(35)}$, and abnormalities in its phenotype and functions have been associated with a variety of autoimmune disorders, including $\mathrm{SLE}^{(36)}$.

Currently, SLE therapy includes corticosteroids and immunosuppressants, with varying success and usually severe side effects $^{(37)}$. For this reason, new therapeutic strategies continue to be investigated and the interest in dietary supplements and nutritional therapy may be considered as a safe therapeutic strategy for SLE patients. Importantly, recent epidemiologic, clinical and experimental studies have suggested that VOO and especially its phenols might possess preventive effect on immune-inflammatory-related diseases, including $\operatorname{SLE}^{(14,15,17,18,21)}$. However, the effects of VOO or its PF on immune-inflammatory functions of the monocyte-macrophage lineage remain to be elucidated.

Oxidative stress plays a substantial role not only in the pathogenesis of autoimmune rheumatic diseases and their complications, but also on organ/system-specific disease activity. Thus, a deregulation of redox homoeostasis may lead to over-production of pro-inflammatory cytokines and NO, and thereby to a condition of oxidative stress, which plays an important role in $\mathrm{SLE}^{(38)}$. Furthermore, the modifications of lipids, proteins and DNA associated with increased iNOS activity have been shown to be involved in the pathogenesis of SLE $^{(39)}$. This highlights that targeted therapies for iNOS and oxidative stress may provide the means to reduce the pathogenic consequences of SLE.

Here we found that a diet containing VOO contributed to prevent $\mathrm{NO}$ and pro-inflammatory cytokine production in peritoneal macrophages of mice with pristane-induced SLE. The amount of VOO administered to animals was equivalent to $20 \mathrm{~g}$ daily consumption for a person of $70 \mathrm{~kg}$ body weight. A lower production of $\mathrm{NO}$ and pro-inflammatory cytokines was also observed in LPS-treated peritoneal macrophages from pristaneinduced SLE mice fed a diet containing VOO when compared with SOD. These observations suggest that VOO may be beneficial for attenuating pristane-induced inflammation in the mouse model of SLE, in agreement with a previous study reporting lower paw swelling, weight of spleen and thymus, urinary level of proteins, blood level of matrix metalloproteinase-3 (MMP-3) and kidney damage in mice with pristane-induced SLE and fed VOO when compared with those fed sunflower oil ${ }^{(18)}$. VOO was a dietary fat rich in oleic acid and minor compounds such as phenols, squalene and triterpenic alcohols, whereas sunflower oil was rich in linoleic acid, sterols and tocopherols. However, the component or components that make VOO different from sunflower oil in the above conceptual framework are still unknown.

On the basis of this interest and owing to the success of VOO against renal injury in mice with pristane-induced SLE ${ }^{(18)}$ and of PF from VOO against joint inflammation in mice with collageninduced arthritis ${ }^{(40)}$ and activation of $\mathrm{T}$ cells from patients with SLE and healthy subjects ${ }^{(21)}$, we focused on effects of PF from VOO on pro-inflammatory mediators and plasticity in human circulating monocytes and monocyte-derived macrophages. Our study demonstrated the ability of PF from VOO to dampen NO production and iNOS protein and gene expression induced by LPS in human peripheral blood monocytes in vitro. These findings show that VOO and its PF can target iNOS activity in myeloid cells from either mice or humans. They are also in good agreement with those previously reported in LPS-treated peritoneal macrophages from mice with acute experimental colitis, where the PF from VOO inhibited the increase of iNOS protein expression through NF- $\kappa \mathrm{B}$ (NF- $\kappa$-light-chain enhancer of activated $\mathrm{B}$ cells) and mitogen-activated protein kinases signalling pathways ${ }^{(16,17)}$.

Modulation of pro-inflammatory mediators in the monocytemacrophage lineage is considered one of the strategies to develop therapeutic compounds against several inflammatory diseases. In this regard, TLR4 plays an important role in monocyte and macrophage activation and in macrophage polarisation by the recognition of LPS, which triggers the release of cytokines with a predominant role in the inflammatory response $^{(41)}$. Our results revealed that PF from VOO dampens TLR4 and pro-inflammatory cytokine gene expression, as well as pro-inflammatory cytokine production induced by LPS in human monocytes in vitro. This provides evidence to suggest that PF from VOO may be effective in reducing LPS-mediated inflammation by interfering with the LPS/TLR4 axis.

Despite the limited available data related to PPAR $\gamma$ and SLE, some studies have shown the potential therapeutic benefits of PPAR $\gamma$ agonists on this disease ${ }^{(42)}$. According to previous studies, the activation of PPAR $\gamma$ may cause an inhibition in the expression of pro-inflammatory cytokines and may drive the differentiation of immune cells to anti-inflammatory phenotypes ${ }^{(43,44)}$. In our study, we found that PF from VOO not only avoided the LPS-induced decreases of PPAR $\gamma$ gene expression but demonstrated its potential in augmenting the transcriptional activity of this gene in human peripheral blood monocytes, which probably contributed to the observed lower inflammatory phenotype after LPS challenge.

Macrophages are key modulator and effector cells in the immune response because their activation influences and responds to other arms of the immune system. In vitro, they can be classified into different subsets, including naïve M0, by the treatment of peripheral blood monocytes with M-CSF and macrophages polarised from M0 by LPS and IFN- $\gamma$ (M1 macrophages, which express a spectrum of pro-inflammatory molecules such as those mentioned above) or by IL-4 (M2 macrophages, which express a wide array of anti-inflammatory molecules $)^{(45)}$. Therefore, we were interested in examining whether PF from VOO had any influence on polarisation of M0 macrophages in terms of their phenotype towards pro- or antiinflammatory direction. It was noteworthy that PF from VOO 
blocked the expression of M1 signature genes and favoured the phenotype of M2 macrophages induced by IL-4, probably suggesting the beneficial influence of PF from VOO on both polarisation channels. The skewing into a subset with an M2like phenotype, even upon treatment with LPS and IFN- $\gamma$, consistently emphasises that PF from VOO may be also beneficial for maintaining tissue homoeostasis under inflammatory conditions. The effects of PF from VOO on LPS-induced deregulation of human peripheral blood monocyte subsets in vitro, by retaining proportions of classical, intermediate and non-classical subsets to values found in untreated cells, further support this notion. A deregulation of peripheral blood monocytes based on an increase in the proportion of the classical subset and a decrease in the proportion of the non-classical subset has been reported in patients with active SLE ${ }^{(46)}$. Our study also found an inhibitory effect of PF from VOO on CCR2 surface and gene expression in LPS-treated human peripheral blood monocytes, which is indicative of a reduction in their migratory capacity and traffic to sites of inflammation ${ }^{(47,48)}$.

The effects of chemicals or diets in animal models are not always predictive for humans. Therefore, it is of crucial importance to investigate the potential benefits of VOO and PF from VOO, the latter as a non-synthetic anti-inflammatory dietary complement, in human disease. Unfortunately, we do not provide data on comparative effects of individual components of $\mathrm{PF}$, appropriate doses or long-term intake of this fraction from $\mathrm{VOO}$ in healthy and/or diseased volunteers, which of course will need to be assessed in future studies. However, for the first time, our study provides several lines of in vivo and in vitro evidence that VOO and PF from VOO target and counteract inflammatory pathways in the monocyte-macrophage lineage of mice with pristane-induced SLE and of healthy subjects, which is a meaningful foundation for further development and application in preclinical and clinical use of PF from VOO in patients with SLE. Thus, we anticipate that VOO, and particularly its PF, can be helpful in reducing SLE activity and be part of the armamentarium in the management of SLE.

\section{Acknowledgements}

M. A.-S. gratefully acknowledges support from a Postgraduate National Program of FPU fellowship and financial sponsorship from the Spanish Ministerio de Educación, Cultura y Deporte. S. M.-d. l. P. has the benefit of a FPI fellowship (BES-2012-056104) of MICINN. B. B. and S. M.-d. 1. P. acknowledge support from 'V Own Research Plan' (University of Seville). The authors gratefully acknowledge the assistance of Centre for Technology and Innovation Research, University of Seville (CITIUS). The authors thank $\mathrm{I}+\mathrm{D}+\mathrm{i}$ of Oleoestepa SAC for kindly providing the VOO.

This study was supported by research grants AGL2011-26949 and AGL2011-29008 (Spanish Ministry of Science and Innovation, MICINN) and P-10AGR-6609 (Junta de Andalucía).

M. A.-S. and S. M.-d. 1. P. performed cell cultures, cytokine measurements and RT-qPCR and western blot experiments. S. M.-d. 1. P. and B. B. performed flow cytometry experiments and analysed the data. M. A.-S., S. M.-d. 1. P. and F. J. G. M. wrote the main manuscript. C. A. d. 1. L., M. S.-H. and F. J. G. M. designed and supervised the project and revised the paper. All authors discussed the results and implications and commented the manuscript at all stages.

The authors declare that there are no conflicts of interest.

\section{Supplementary material}

For supplementary material/s referred to in this article, please visit https://doi.org/10.1017/S0007114518001976

\section{References}

1. Helmick CG, Felson DT, Lawrence RC, et al. (2008) Estimates of the prevalence of arthritis and other rheumatic conditions in the United States. Part I. Arthritis Rheum 58, 15-25.

2. Peng SL (2009) Altered T and B lymphocyte signaling pathways in lupus. Autoimmun Rev 8, 179-183.

3. Geissmann F, Manz MG, Jung S, et al. (2010) Development of monocytes, macrophages, and dendritic cells. Science 327, 656-661.

4. Tugal D, Liao X \& Jain MK (2013) Transcriptional control of macrophage polarization. Arterioscler Thromb Vasc Biol 33, 1135-1144

5. Kavai M \& Szegedi G (2007) Immune complex clearance by monocytes and macrophages in systemic lupus erythematosus. Autoimmun Rev 6, 497-502.

6. Rönnblom L, Eloranta ML \& Alm GV (2006) The type I interferon system in systemic lupus erythematosus. Arthritis Rheum 54, 408-420.

7. Sestak AL, Fürnrohr BG, Harley JB, et al. (2011) The genetics of systemic lupus erythematosus and implications for targeted therapy. Ann Rbeum Dis 70, Suppl. 1, i37-i43.

8. Satoh M \& Reeves WH (1994) Induction of lupus-associated autoantibodies in BALB/c mice by intraperitoneal injection of pristane. J Exp Med 180, 2341-2346.

9. Satoh M, Yamagata H, Watanabe F, et al. (1995) Development of anti-Sm and anti-DNA antibodies followed by clinical manifestation of systemic lupus erythematosus in an elderly woman with long-standing Sjögren's syndrome. Lupus 4, 63-65.

10. Shaheen VM, Satoh M, Richards HB, et al. (1999) Immunopathogenesis of environmentally induced lupus in mice. Environ Health Perspect 107, Suppl. 5, 723-727.

11. De Rosa V, Galgani M, Santopaolo M, et al. (2015) Nutritional control of immunity: balancing the metabolic requirements with an appropriate immune function. Semin Immunol 27, 300-309.

12. Aparicio-Soto M, Sanchez-Hidalgo M \& Alarcon-de-la-Lastra C (2017) An update on diet and nutritional factors in systemic lupus erythematosus management. Nutr Res Rev 30, 118-137.

13. Buckland G, Mayen AL, Agudo A, et al. (2012) Olive oil intake and mortality within the Spanish population (EPIC-Spain). Am J Clin Nutr 96, 142-149.

14. Alarcon de la Lastra C, Barranco MD, Motilva V, et al. (2001) Mediterranean diet and health: biological importance of olive oil. Curr Pharm Des 7, 933-950.

15. Cardeno A, Sanchez-Hidalgo M \& Alarcon-de-la-Lastra C (2013) An up-date of olive oil phenols in inflammation and cancer: molecular mechanisms and clinical implications. Curr Med Chem 20, 4758-4776.

16. Cardeno A, Sanchez-Hidalgo M, Aparicio-Soto M, et al. (2014) Extra virgin olive oil polyphenolic extracts downregulate inflammatory responses in LPS-activated murine peritoneal macrophages suppressing NF kappa B and MAPK signalling pathways. Food Funct 5, 1270-1277. 
17. Sanchez-Fidalgo S, Cardeno A, Sanchez-Hidalgo M, et al. (2013) Dietary extra virgin olive oil polyphenols supplementation modulates DSS-induced chronic colitis in mice. J Nutr Biochem 24, 1401-1413.

18. Aparicio-Soto M, Sanchez-Hidalgo M, Cardeno A, et al. (2016) Dietary extra virgin olive oil attenuates kidney injury in pristane-induced SLE model via activation of $\mathrm{HO}-1 / \mathrm{Nrf}-2$ antioxidant pathway and suppression of JAK/STAT, NF- $\mathrm{B}$ and MAPK activation. J Nutr Biochem 27, 278-288.

19. Rosillo MA, Sanchez-Hidalgo M, Sanchez-Fidalgo S, et al. (2016) Dietary extra-virgin olive oil prevents inflammatory response and cartilage matrix degradation in murine collageninduced arthritis. Eur J Nutr 5, 315-325.

20. Sanchez-Fidalgo S, Villegas I, Cardeno A, et al. (2010) Extravirgin olive oil-enriched diet modulates DSS-colitis-associated colon carcinogenesis in mice. Clin Nutr 29, 663-673.

21. Aparicio-Soto M, Sanchez-Hidalgo M, Cardeno A, et al. (2017) The phenolic fraction of extra virgin olive oil modulates the activation and the inflammatory response of $\mathrm{T}$ cells from patients with systemic lupus erythematosus and healthy donors. Mol Nutr Food Res 61, 1-8.

22. Aparicio-Soto M, Alarcon-de-la-Lastra C, Cardeno A, et al. (2014) Melatonin modulates microsomal PGE synthase 1 and NF-E2-related factor-2-regulated antioxidant enzyme expression in LPS-induced murine peritoneal macrophages. $\mathrm{Br} J$ Pharmacol 171, 134-144.

23. Kilkenny C, Browne WJ, Cuthill IC, et al. (2010) Improving bioscience research reporting: the ARRIVE guidelines for reporting animal research. J Pharmacol Pharmacother 1, 94-99.

24. McGrath JC, Drummond GB, McLachlan EM, et al. (2010) Guidelines for reporting experiments involving animals: the ARRIVE guidelines. Br J Pharmacol 160, 1573-1576.

25. Moorcroft MJ, Davis J \& Compton RG (2001) Detection and determination of nitrate and nitrite: a review. Talanta $\mathbf{5 4}$, 785-803.

26. Vazquez Roncero A, Janet del Valle M \& Janet del Valle L (1976) Componentes fenolicos de la aceituna. III, Polifenoles del aceite (Phenolic compounds from olives. III, Oil polyphenols). Grasas aceites (B Aires) 27, 185-191.

27. Denizot F \& Lang R (1986) Rapid colorimetric assay for cell growth and survival. Modifications to the tetrazolium dye procedure giving improved sensitivity and reliability. J Immunol Methods 89, 271-277.

28. Sanchez-Hidalgo M, Martin AR, Villegas I, et al. (2005) Rosiglitazone, an agonist of peroxisome proliferator-activated receptor gamma, reduces chronic colonic inflammation in rats. Biochem Pharmacol 69, 1733-1744.

29. Bradford MM (1976) A rapid and sensitive method for the quantitation of microgram quantities of protein utilizing the principle of protein-dye binding. Anal Biochem $\mathbf{7 2}$, 248-254.

30. Feng GJ, Goodridge HS, Harnett MM, et al. (1999) Extracellular signal-related kinase (ERK) and p38 mitogen-activated protein (MAP) kinases differentially regulate the lipopolysaccharide-mediated induction of inducible nitric oxide synthase and IL-12 in macrophages: Leishmania phosphoglycans subvert macrophage IL-12 production by targeting ERK MAP kinase. J Immunol 163, 6403-6412.
31. Chow JC, Young DW, Golenbock DT, et al. (1999) Toll-like receptor-4 mediates lipopolysaccharide-induced signal transduction. J Biol Chem 274, 10689-10692.

32. Cole JE, Georgiou E \& Monaco C (2010) The expression and functions of Toll-like receptors in atherosclerosis. Mediators Inflamm 2010, 393946.

33. Ziegler-Heitbrock L (2014) Monocyte subsets in man and other species. Cell Immunol 289, 135-139.

34. Li Y, Lee PY \& Reeves WH (2010) Monocyte and macrophage abnormalities in systemic lupus erythematosus. Arch Immunol Ther Exp (Warsz) 58, 355-364.

35. Unanue ER (1978) The regulation of lymphocyte functions by the macrophage. Immunol Rev 40, 227-255.

36. Katsiari CG, Liossis SN \& Sfikakis PP (2010) The pathophysiologic role of monocytes and macrophages in systemic lupus erythematosus: a reappraisal. Semin Arthritis Rheum 39, 491-503.

37. van Vollenhoven RF, Parodis I \& Levitsky A (2013) Biologics in SLE: towards new approaches. Best Pract Res Clin Rbeumatol 27, 341-349.

38. Sukkar SG \& Rossi E (2004) Oxidative stress and nutritional prevention in autoimmune rheumatic diseases. Autoimmun Rev 3, 199-206.

39. Oates JC \& Gilkeson GS (2006) The biology of nitric oxide and other reactive intermediates in systemic lupus erythematosus. Clin Immunol 121, 243-250.

40. Rosillo MA, Alcaraz MJ, Sanchez-Hidalgo M, et al. (2014) Anti-inflammatory and joint protective effects of extra-virgin olive-oil polyphenol extract in experimental arthritis. $J$ Nutr Biochem 25, 1275-1281.

41. Chen L, Yang S, Zumbrun EE, et al. (2015) Resveratrol attenuates lipopolysaccharide-induced acute kidney injury by suppressing inflammation driven by macrophages. Mol Nutr Food Res 59, 853-864.

42. Venegas-Pont M, Sartori-Valinotti JC, Maric C, et al. (2009) Rosiglitazone decreases blood pressure and renal injury in a female mouse model of systemic lupus erythematosus. Am J Physiol Regul Integr Comp Physiol 296, R1282-R1289.

43. Chinetti G, Fruchart JC \& Staels B (2000) Peroxisome proliferator-activated receptors (PPARs): nuclear receptors at the crossroads between lipid metabolism and inflammation. Inflamm Res 49, 497-505.

44. Martin H (2009) Role of PPAR-gamma in inflammation. Prospects for therapeutic intervention by food components. Mutat Res 669, 1-7.

45. Perez-Jimenez F, Alvarez de Cienfuegos G, Badimon L, et al. (2005) International conference on the healthy effect of virgin olive oil. Eur J Clin Invest 35, 421-424.

46. Burbano C, Vasquez G \& Rojas M (2014) Modulatory effects of $\mathrm{CD} 14^{+} \mathrm{CD} 16^{++}$monocytes on $\mathrm{CD} 14^{++} \mathrm{CD} 16^{-}$monocytes: a possible explanation of monocyte alterations in systemic lupus erythematosus. Arthritis Rheumatol 66, 33713381.

47. Moser KL, Kelly JA, Lessard CJ, et al. (2009) Recent insights into the genetic basis of systemic lupus erythematosus. Genes Immun 10, 373-379.

48. Yang J, Zhang L, Yu C, et al. (2014) Monocyte and macrophage differentiation: circulation inflammatory monocyte as biomarker for inflammatory diseases. Biomark Res $\mathbf{2}, 1$. 